\title{
Fine-Tuning Contextual-Based Optimum-Path Forest for Land-Cover Classification
}

\author{
Daniel Osaku, Danillo R. Pereira, Alexandre L. M. Levada, and João P. Papa
}

\begin{abstract}
Contextual-based learning aims at considering neighboring pixels to improve pixelwise-oriented classification techniques. In this letter, we presented a metaheuristic framework for the optimization of nondiscrete Markovian models considering the optimum-path forest (OPF) classifier, and we proposed a postprocessing procedure to avoid overcorrection over high-frequency regions. The proposed approach outperformed previous results obtained with standard OPF in satellite imagery.
\end{abstract}

Index Terms-Contextual classification, optimum-path forest (OPF).

\section{INTRODUCTION}

$\mathbf{C}$ ONTEXTUAL-based classification has been widely employed in a wide range of applications. Schmidt et al. [1], for instance, combined conditional random fields and random forests for airborne data classification, and Tarabalka et al. [2] proposed a hybrid approach based on support vector machines (SVMs) and Markov random field (MRF) called SVMMRF for contextual-based remote sensing image classification. Furthermore, Moser and Serpico [3] proposed a single-step version of the SVM-MRF classifier, rather than the two-phase strategy presented in [2].

Later on, Ghamisi et al. [4] addressed contextual classification of hyperspectral images using SVM and hidden Markov random fields. Although the SVM classifier has drawn a considerable attention out there, it may suffer from a high computational burden for fine-tuning the kernel parameters. Such shortcoming has led researchers to focus on alternatives, such as lightweight techniques or even ensemble of weak classifiers. Papa et al. [5], [6] proposed a graph-based classifier named optimum-path forest (OPF), which has demonstrated to be similar to SVM, but much faster and easier to be used, since it is parameterless and does not make assumptions about the separability of samples and their distributions.

The OPF classifier has been successfully employed in remotesensing-oriented applications [7], and its contextual version

Manuscript received February 26, 2015; revised July 23, 2015 and December 18, 2015; accepted March 6, 2016. Date of publication April 4, 2016; date of current version April 20, 2016. This work was supported in part by The São Paulo Research Foundation (FAPESP) under Grant 2012/06472-9, Grant 2013/20387-7, and Grant 2014/16250-9 and in part by the National Council for Scientific and Technological Development (CNPq) of Brazil under Grant 303182/2011-3, Grant 470571/2013-6, and Grant 306166/2014-3.

D. Osaku and A. L. M. Levada are with the Department of Computer Science, Federal University of São Carlos, 13565-905 São Carlos, Brazil (e-mail: danosaku@hotmail.com; alexandre@dc.ufscar.br).

D. R. Pereira and J. P. Papa are with the Department of Computing, São Paulo State University, 01049-010 São Paulo, Brazil (e-mail: dpereira@ic.unicamp. br; papa@fc.unesp.br).

Color versions of one or more of the figures in this paper are available online at http://ieeexplore.ieee.org.

Digital Object Identifier 10.1109/LGRS.2016.2541458 called OPF-MRF makes use of MRF theory to consider neighboring information [8]. Since OPF-MRF employs the PottsIsing formulation to model the spatial dependence among nearby pixels, Osaku et al. [9] further proposed a metaheuristic-based approach to fine-tune the amount of spatial information used during the classification process, and Osaku et al. [10] compared different hand-tuned Markovian models concerning OPFMRF for land-cover image classification. A deeper evaluation of OPF-MRF for land-use classification can be found in [8].

In this letter, we extend the work of Osaku et al. [10] by automatic fine-tuning a number of Markovian models for contextual land-use classification in satellite imagery using metaheuristic techniques. Additionally, this letter also shows that we can improve naïve OPF-MRF classification results using a mode filter applied under some conditions to avoid overcorrections over high-frequency regions. The remainder of this letter is organized as follows. Sections II and III present the proposed approach and the methodology, respectively. Experimental results are presented in Section IV, and Section V states conclusions.

\section{Fine-Tuning Contextual OPF THROUGH Metaheuristics}

Initially, the former versions of OPF-MRF employed the well-known Potts model to encode the spatial content around nearby samples. Basically, given a neighborhood system $\eta_{i j}$ with respect to a central pixel $(i, j)$, we can define the local conditional probability of Potts model as follows:

$$
p\left(l_{i j}=m \mid \eta_{i j}, \beta\right)=\frac{\exp \left\{\beta \mathcal{U}_{i j}(m)\right\}}{\sum_{k=1}^{K} \exp \left\{\beta \mathcal{U}_{i j}(k)\right\}}
$$

in which $\mathcal{U}_{i j}(k)$ stands for the number of pixels in $\eta_{i j}$ that are from label $k, K$ denotes the number of labels, $\beta$ is a parameter that models the spatial dependence among neighboring pixels, and $m$ means the observed label at central pixel $(i, j) .{ }^{1}$

The metaheuristic-oriented technique to fine-tune the MRF models used in this letter is based on the work presented by Osaku et al. [8], [9]. However, we extended their approach to different Markovian models that are not discrete-oriented since the aforementioned works applied the fine-tuning approach for the Potts model only, which has been designed to work over discrete MRF models (samples' label). Therefore, such model is expected to work better than continuous-based ones. In this letter, we show that both discrete and nondiscrete models can obtain similar accuracy when fine-tuned by some optimization technique.

\footnotetext{
${ }^{1}$ Notice that high values of $\beta$ lead to high spatial dependence among pixels, and $\beta=0$ means we have no spatial dependence.
} 


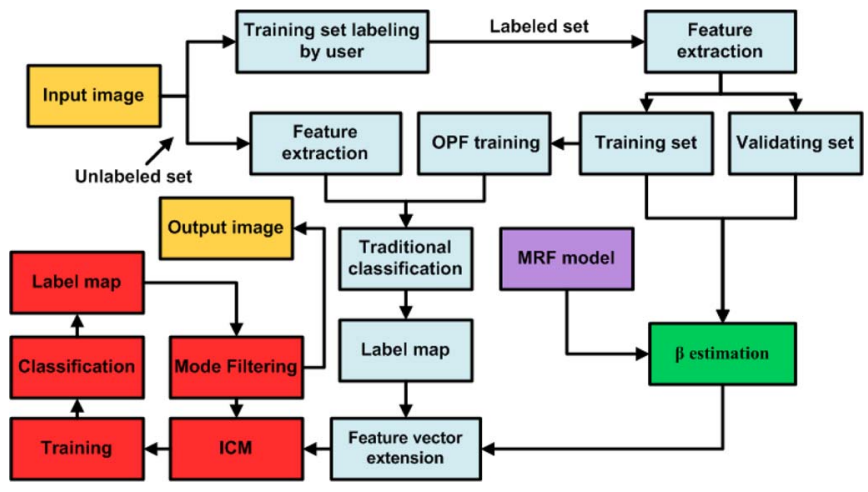

Fig. 1. Proposed pipeline for model-independent OPF-MRF.

The OPF-MRF models the contextual information in three steps: 1) first, a traditional classification is carried out by naïve OPF; 2) then, the labeled map generated in the previous step is used to augment the feature vector of each sample with its neighborhood information, and the learning process followed by classification is then executed again by OPF; and 3) finally, the iterated conditional mode (ICM) algorithm is used over the label map to maximize the probability $p(\mathcal{Y} \mid \mathcal{X})$, in which $\mathcal{Y}$ and $\mathcal{X}$ stand for the whole random field of class labels and feature vectors, respectively. Steps 2 and 3 are then repeated over again along $T$ iterations, but now using the image obtained after the ICM algorithm as the input to the standard OPF at step 2.

Based on the approach introduced by Osaku et al. [8], [9], we extended the fine-tuning parameters to others MRF models. The idea is to use OPF-MRF with a third set of samples hereinafter called validation set. The accuracy of OPF-MRF over that set is then used as the fitness function to guide the optimization techniques. Fig. 1 shows the proposed pipeline, being the "green" module the one in charge of the MRF finetuning parameter procedure.

However, we may still have misclassified pixels in areas composed of small connected components surrounded by samples from different classes. In this letter, we proposed a simple but effective approach to deal with this problem: We employed a mode filter as a postprocessing step, but only when a predefined criterion is met since we would like to avoid overcorrections over high-frequency regions and thus may degrade the previous results. The criterion is performed by computing the frequency of each label within a $3 \times 3$ window centered at a given pixel, for further defining the most frequent label $l$. A mode value of $M$ means we applied the mode filter over that window if $n>M$, where $n$ stands for the number of pixels classified with label $l .^{2}$

\section{Methodology}

In this letter, we employed two remote sensing images obtained from CBERS-2B satellite covering the area of Itatinga, SP-Brazil, and another image obtained from Ikonos-2 MS satellite covering the area of Duque de Caxias, RJ-Brazil. Fig. 2 shows such images and their corresponding ground-truth versions. ${ }^{3}$

\footnotetext{
${ }^{2}$ In this letter, we used $M \in\{3,4,5\}$ for a window size of $3 \times 3$, and $M \in$ $\{3, \ldots, 15\}$ for a window size of $5 \times 5$. However, due to the lack of space, we displayed the values for $M \in\{3,4\}$ with a windows size of $3 \times 3$ only since they have obtained the best results.

${ }^{3}$ The reader can refer to the work conducted by Osaku et al. [8] for a more detailed explanation about the data sets.
}

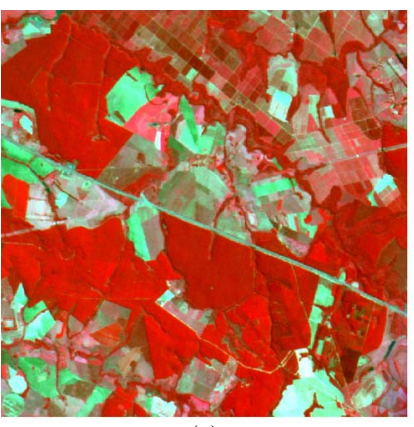

(a)

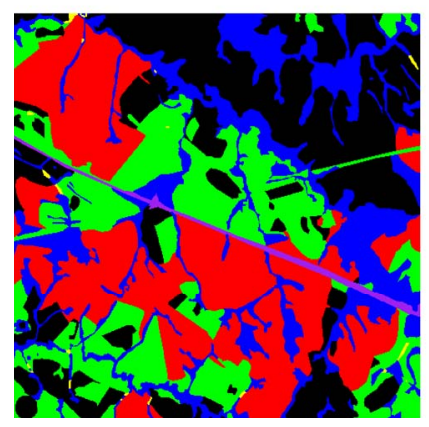

(c)

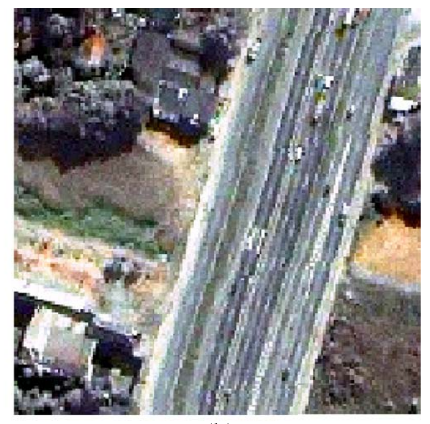

(b)

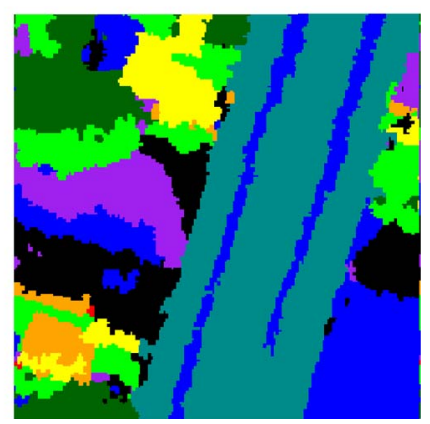

(d)
Fig. 2. Images used in the experiments. (a) Covering the area of Itatinga, SPBrazil, by CBERS-2B CCD $(20 \mathrm{~m})$ sensor (R2G3B4). (b) Covering the area of Duque de Caxias, RJ-Brazil, by Ikonos-2 MS sensor (R4G3B2). CBERS-2B and Ikonos-2 MS ground-truth images are displayed in (c) and (d), respectively.

TABLE I

MEan ACCURACy Rates Using DifFEREnT MRF Models OVER CBERS2-B IMAGE WITH FEATURE EXTRACTION ALGORITHMS (I) AND (II)

\begin{tabular}{|l|c|c|}
\hline Technique & (I) & (II) \\
\hline OPF (baseline) & $87.04 \% \pm 0.41$ & $87.45 \% \pm 0.37$ \\
OPF-MRF/Potts & $\mathbf{9 0 . 0 8 \%} \pm \mathbf{0 . 5 4}$ & $\mathbf{9 0 . 8 5 \%} \pm \mathbf{0 . 2 9}$ \\
OPF-MRF/GIMLL-L1 & $\mathbf{8 9 . 5 2 \%} \pm \mathbf{0 . 3 3}$ & $\mathbf{9 0 . 4 4 \%} \pm \mathbf{0 . 4 3}$ \\
OPF-MRF/GIMLL-L2 & $\mathbf{8 9 . 7 5 \%} \pm \mathbf{0 . 4 0}$ & $\mathbf{9 0 . 3 9 \%} \pm \mathbf{0 . 4 0}$ \\
OPF-MRF/GMRF & $\mathbf{8 9 . 4 1 \%} \pm \mathbf{0 . 4 4}$ & $\mathbf{8 9 . 6 9 \%} \pm \mathbf{0 . 2 8}$ \\
\hline SVM (baseline) & $87.35 \% \pm 0.32$ & $87.93 \% \pm 0.26$ \\
SVM-MRF/Potts & $\mathbf{8 9 . 7 3 \%} \pm \mathbf{0 . 3 5}$ & $90.36 \% \pm 0.23$ \\
SVM-MRF/GIMLL-L1 & $\mathbf{8 9 . 6 1 \%} \pm \mathbf{0 . 2 1}$ & $90.28 \% \pm 0.47$ \\
SVM-MRF/GIMLL-L2 & $\mathbf{8 9 . 6 7 \%} \pm \mathbf{0 . 2 4}$ & $90.06 \% \pm 0.35$ \\
SVM-MRF/GMRF & $89.00 \% \pm 0.28$ & $89.34 \% \pm 0.20$ \\
\hline
\end{tabular}

TABLE II

Mean ACCuracy Rates Using Different MRF Models OVER IKONOS-2 MS IMAGE With FEATURE EXTRACTION ALGORITHMS (I) AND (II)

\begin{tabular}{|l|c|c|}
\hline Technique & (I) & (II) \\
\hline OPF (baseline) & $78.83 \% \pm 0.35$ & $85.87 \% \pm 0.42$ \\
OPF-MRF/Potts & $\mathbf{8 5 . 5 0 \%} \pm \mathbf{0 . 4 5}$ & $\mathbf{8 8 . 6 3 \%} \pm \mathbf{0 . 7 0}$ \\
OPF-MRF/GIMLL-L1 & $81.92 \% \pm 0.51$ & $\mathbf{8 7 . 6 5 \%} \pm \mathbf{0 . 4 5}$ \\
OPF-MRF/GIMLL-L2 & $81.40 \% \pm 0.54$ & $\mathbf{8 7 . 8 7 \%} \pm \mathbf{0 . 4 8}$ \\
OPF-MRF/GMRF & $81.26 \% \pm 0.39$ & $87.38 \% \pm 0.58$ \\
\hline SVM (baseline) & $81.79 \% \pm 0.15$ & $\mathbf{8 6 . 9 2} \% \pm \mathbf{0 . 3 6}$ \\
SVM-MRF/Potts & $85.50 \% \pm 0.46$ & $\mathbf{8 7 . 8 8 \%} \pm \mathbf{0 . 6 0}$ \\
SVM-MRF/GIMLL-L1 & $84.31 \% \pm 0.33$ & $\mathbf{8 7 . 3 7 \%} \pm \mathbf{0 . 4 7}$ \\
SVM-MRF/GIMLL-L2 & $84.64 \% \pm 0.50$ & $\mathbf{8 7 . 6 3 \%} \pm \mathbf{0 . 5 7}$ \\
SVM-MRF/GMRF & $83.58 \% \pm 0.13$ & $\mathbf{8 7 . 0 5} \% \pm \mathbf{0 . 5 8}$ \\
\hline
\end{tabular}

The experiments were conducted as follows: For each satellite image, we have employed 5\% of the pixels to compose the training set, $15 \%$ for the evaluating set, and the remaining $80 \%$ samples to compose the test set. Each pixel is described by two distinct representations: (I) 26 features provided by the RGB 
TABLE III

MeAn ACCuracy Results Considering Different OPF-BASEd Classifiers AND MARKov Models Over CBERS-2B IMAGE With $\beta$ Optimized by BF, HS, and PSO. The Best Accuracy of Each Pair (Markov Model, Optimization TechniQue) Is in Bold

\begin{tabular}{|l|c|c|c|c|c|c|}
\hline Technique & Mode value & BF & HS & PSO & NM & GD \\
\hline OPF & \multicolumn{4}{|c|}{$87.42 \% \pm 0.37$} \\
\hline & - & $\mathbf{9 0 . 9 0 \%} \pm \mathbf{0 . 4 0}$ & $\mathbf{9 0 . 8 2 \%} \pm \mathbf{0 . 2 2}$ & $\mathbf{9 0 . 8 5 \%} \pm \mathbf{0 . 3 3}$ & $\mathbf{9 0 . 7 4 \%} \pm \mathbf{0 . 6 1}$ & $\mathbf{9 0 . 7 2 \%} \pm \mathbf{0 . 5 6}$ \\
OPF-MRF/ & 3 & $88.03 \% \pm 0.45$ & $88.03 \% \pm 0.44$ & $88.05 \% \pm 0.43$ & $87.88 \% \pm 0.56$ & $87.88 \% \pm 0.56$ \\
Potts & 4 & $\mathbf{9 1 . 0 8 \%} \pm \mathbf{0 . 3 5}$ & $\mathbf{9 1 . 0 0 \%} \pm \mathbf{0 . 3 5}$ & $\mathbf{9 0 . 9 0 \%} \pm \mathbf{0 . 4 2}$ & $\mathbf{9 0 . 9 9 \%} \pm \mathbf{0 . 5 7}$ & $\mathbf{9 0 . 9 8 \%} \pm \mathbf{0 . 9 1}$ \\
\hline & - & $\mathbf{9 0 . 3 8 \%} \pm \mathbf{0 . 3 9}$ & $\mathbf{9 0 . 3 3 \%} \pm \mathbf{0 . 4 5}$ & $\mathbf{9 0 . 3 2 \%} \pm \mathbf{0 . 4 9}$ & $\mathbf{9 0 . 3 6 \%} \pm \mathbf{0 . 4 2}$ & $\mathbf{9 0 . 2 1 \%} \pm \mathbf{0 . 5 5}$ \\
OPF-MRF/ & 3 & $87.79 \% \pm 0.40$ & $87.77 \% \pm 0.41$ & $87.78 \% \pm 0.39$ & $87.71 \% \pm 0.43$ & $87.74 \% \pm 0.43$ \\
GIMLL-L1 & 4 & $\mathbf{9 0 . 7 9 \%} \pm \mathbf{0 . 4 2}$ & $\mathbf{9 0 . 7 5 \%} \pm \mathbf{0 . 4 5}$ & $\mathbf{9 0 . 7 7 \%} \pm \mathbf{0 . 4 4}$ & $\mathbf{9 0 . 7 9 \%} \pm \mathbf{0 . 4 6}$ & $\mathbf{9 0 . 7 7 \%} \pm \mathbf{0 . 5 1}$ \\
\hline & - & $\mathbf{9 0 . 3 3 \%} \pm \mathbf{0 . 2 5}$ & $\mathbf{9 0 . 3 3 \%} \pm \mathbf{0 . 3 8}$ & $\mathbf{9 0 . 3 5 \%} \pm \mathbf{0 . 4 3}$ & $\mathbf{9 0 . 3 4 \%} \pm \mathbf{0 . 3 1}$ & $\mathbf{9 0 . 1 9 \%} \pm \mathbf{0 . 4 2}$ \\
OPF-MRF/ & 3 & $87.81 \% \pm 0.47$ & $87.82 \% \pm 0.38$ & $87.78 \% \pm 0.40$ & $87.69 \% \pm 0.45$ & $87.74 \% \pm 0.51$ \\
GIMLL-L2 & 4 & $\mathbf{9 0 . 8 2 \%} \pm \mathbf{0 . 4}$ & $\mathbf{9 0 . 8 0 \%} \pm \mathbf{0 . 5 0}$ & $\mathbf{9 0 . 8 0 \%} \pm \mathbf{0 . 4 6}$ & $\mathbf{9 0 . 8 1 \%} \pm \mathbf{0 . 5 2}$ & $\mathbf{9 0 . 8 1 \%} \pm \mathbf{0 . 4 1}$ \\
\hline & - & $89.64 \% \pm 0.31$ & $\mathbf{8 9 . 7 1 \%} \pm \mathbf{0 . 3 2}$ & $\mathbf{8 9 . 6 9 \%} \pm \mathbf{0 . 3 3}$ & $89.61 \% \pm 0.52$ & $89.48 \% \pm 0.58$ \\
OPF-MRF/ & 3 & $87.71 \% \pm 0.46$ & $87.68 \% \pm 0.45$ & $87.70 \% \pm 0.43$ & $87.66 \% \pm 0.51$ & $87.60 \% \pm 0.52$ \\
GMRF & 4 & $\mathbf{9 0 . 2 6 \%} \pm \mathbf{0 . 4 4}$ & $\mathbf{9 0 . 3 0 \%} \pm \mathbf{0 . 4 3}$ & $\mathbf{9 0 . 3 1 \%} \pm \mathbf{0 . 4 3}$ & $\mathbf{9 0 . 3 7 \%} \pm \mathbf{0 . 5 9}$ & $\mathbf{9 0 . 1 4 \%} \pm \mathbf{0 . 5 7}$ \\
\hline
\end{tabular}

TABLE IV

Mean ACCuRacy Results Considering DifFerent OPF-Based Classifiers and Markov Models Over IKONOS-2 MS IMAGe With $\beta$ Optimized by BF, HS, and PSO. The Best ACCuracy of Each Pair (Markov Model, Optimization TeChNiQue) Is IN Bold

\begin{tabular}{|l|c|c|c|c|c|c|}
\hline Technique & Mode value & BF & HS & PSO & NM & GD \\
\hline OPF & & \multicolumn{5}{|c|}{$85.87 \% \pm 0.42$} \\
\hline & - & $\mathbf{8 8 . 6 5 \%} \pm \mathbf{0 . 6 7}$ & $\mathbf{8 8 . 5 1 \%} \pm \mathbf{0 . 6 1}$ & $\mathbf{8 8 . 5 1 \%} \pm \mathbf{0 . 6 7}$ & $\mathbf{8 8 . 3 4 \%} \pm \mathbf{0 . 5 8}$ & $\mathbf{8 8 . 3 4 \%} \pm \mathbf{0 . 6 3}$ \\
OPF-MRF/ & 3 & $\mathbf{8 8 . 0 0 \%} \pm \mathbf{0 . 4 0}$ & $\mathbf{8 8 . 1 3 \%} \% \mathbf{0 . 3 6}$ & $\mathbf{8 8 . 0 6 \%} \pm \mathbf{0 . 4 0}$ & $\mathbf{8 8 . 0 0 \%} \pm \mathbf{0 . 3 6}$ & $\mathbf{8 7 . 9 9 \%} \pm \mathbf{0 . 3 8}$ \\
Potts & 4 & $\mathbf{8 8 . 8 8 \%} \pm \mathbf{0 . 6 1}$ & $\mathbf{8 9 . 0 0 \%} \pm \mathbf{0 . 6 3}$ & $\mathbf{8 8 . 9 6 \%} \pm \mathbf{0 . 6 7}$ & $\mathbf{8 8 . 7 3 \%} \pm \mathbf{0 . 4 0}$ & $\mathbf{8 8 . 8 9 \%} \pm \mathbf{0 . 6 1}$ \\
\hline & - & $87.87 \% \pm 0.53$ & $87.63 \% \pm 0.40$ & $87.66 \% \pm 0.47$ & $87.77 \% \pm 0.47$ & $87.70 \% \pm 0.62$ \\
OPF-MRF/ & 3 & $\mathbf{8 8 . 0 1 \%} \pm \mathbf{0 . 4 8}$ & $\mathbf{8 8 . 0 2 \%} \pm \mathbf{0 . 4 1}$ & $\mathbf{8 8 . 0 4 \%} \pm \mathbf{0 . 4 3}$ & $\mathbf{8 7 . 9 4 \%} \pm \mathbf{0 . 4 0}$ & $\mathbf{8 8 . 0 2 \%} \pm \mathbf{0 . 3 7}$ \\
GIMLL-L1 & 4 & $\mathbf{8 8 . 7 7 \%} \pm \mathbf{0 . 3 9}$ & $\mathbf{8 8 . 8 8 \%} \pm \mathbf{0 . 5 5}$ & $\mathbf{8 8 . 8 7 \%} \pm \mathbf{0 . 4 4}$ & $\mathbf{8 8 . 8 2 \%} \pm \mathbf{0 . 1 2}$ & $\mathbf{8 8 . 7 9 \%} \pm \mathbf{0 . 4 1}$ \\
\hline & - & $87.88 \% \pm 0.53$ & $\mathbf{8 7 . 9 3 \%} \pm \mathbf{0 . 5 0}$ & $87.90 \% \pm 0.47$ & $\mathbf{8 8 . 1 1 \%} \pm \mathbf{0 . 6 4}$ & $\mathbf{8 7 . 9 8 \%} \pm \mathbf{0 . 6 1}$ \\
OPF-MRF/ & 3 & $\mathbf{8 8 . 0 3 \%} \pm \mathbf{0 . 4 9}$ & $\mathbf{8 8 . 0 2 \%} \% \mathbf{0 . 5 0}$ & $\mathbf{8 8 . 0 3 \%} \pm \mathbf{0 . 4 9}$ & $\mathbf{8 7 . 9 9 \%} \pm \mathbf{0 . 3 5}$ & $\mathbf{8 7 . 9 9 \%} \pm \mathbf{0 . 3 8}$ \\
GIMLL-L2 & 4 & $\mathbf{8 9 . 0 9 \%} \pm \mathbf{0 . 5 8}$ & $\mathbf{8 9 . 0 0 \%} \pm \mathbf{0 . 6 9}$ & $\mathbf{8 9 . 0 2 \%} \pm \mathbf{0 . 5 1}$ & $\mathbf{8 8 . 9 8 \%} \pm \mathbf{0 . 4 7}$ & $\mathbf{8 9 . 0 2 \%} \pm \mathbf{0 . 5 0}$ \\
\hline & - & $87.40 \% \pm 0.49$ & $87.02 \% \pm 0.97$ & $87.07 \% \pm 0.97$ & $86.63 \% \pm 1.18$ & $86.13 \% \pm 1.27$ \\
OPF-MRF/ & 3 & $\mathbf{8 8 . 1 6 \%} \pm \mathbf{0 . 4 1}$ & $\mathbf{8 8 . 1 0 \%} \pm \mathbf{0 . 3 8}$ & $\mathbf{8 8 . 1 4 \%} \pm \mathbf{0 . 3 8}$ & $\mathbf{8 7 . 9 4 \%} \pm \mathbf{0 . 3 7}$ & $\mathbf{8 7 . 9 0 \%} \pm \mathbf{0 . 4 0}$ \\
GMRF & 4 & $\mathbf{8 8 . 8 6 \%} \pm \mathbf{0 . 5 7}$ & $\mathbf{8 8 . 6 6 \%} \pm \mathbf{0 . 5 6}$ & $\mathbf{8 8 . 7 2 \%} \pm \mathbf{0 . 5 4}$ & $\mathbf{8 8 . 5 6 \%} \pm \mathbf{0 . 3 6}$ & $\mathbf{8 8 . 2 2 \%} \pm \mathbf{0 . 4 2}$ \\
\hline
\end{tabular}

values of its eight-neighborhood ${ }^{4}$ and the pixel position $(x, y)$; and (II) the other one is composed of five features provided by its RGB values and the pixel position $(x, y)$. Additionally, we performed a ten-cross-validation procedure for the analysis of experiments. To show that the proposed approach can be applied to other classifiers, we also considered SVM with a radial basis function in the experimental evaluation. ${ }^{5}$

For each Markov model, we employed the particle swarm optimization (PSO), harmony search (HS), gradient descent (GD), and the Nelder-Mead (NM) to find out suitable values for $\beta$, being the baseline for such comparison a near-exhaustive search, hereinafter called the brute-force (BF) method. ${ }^{6}$ We used ten iterations and five particles for PSO, 50 iterations and five harmonies for HS, and 50 iterations for BF. Furthermore, we have used $T=10$ iterations considering OPF-MRF for both optimization and classification steps. Finally, for PSO, we have used $w=0.5$ and $c_{1}=c_{2}=2$, and considering HS, we used $\mathrm{HMCR}=0.9$ and $\mathrm{PAR}=0.5 .^{7}$

\section{Simulations And Results}

The first experiment aims at comparing the two feature extraction techniques, i.e., the one which considers a

\footnotetext{
${ }^{4} \mathrm{We}$ are not considering the RGB values of the central pixel in this letter.

${ }^{5} \mathrm{SVM}$ parameters were estimated through cross-validation.

${ }^{6}$ The search range for PSO, HS, NM, and GD is $\beta \in\left[0, \beta_{\max }\right]$ [8], where $\beta_{\max }=\ln (1+\sqrt{K})$. The same search range is applied for BF, but with steps of $\beta_{\max } / 50$.

${ }^{7}$ Recall that all parameter values were based on our previous works [8]-[10]. Such values were empirically chosen by following some guidelines usually employed by related literature.
}

26-dimensional feature vector (I), and the another composition that employs five features (II). Tables I and II present the results over CBERS-2B and Ikonos-2 MS images, respectively, with the most accurate results in bold.

This first experiment pointed out some results not observed by the work of Osaku et al. [10]: In their previous work, a handtuning approach is used to select the most suitable values for the MRF models, with the best one being the Potts model. The authors stated that such results were very reasonable since Potts model was designed to address MRFs composed by discrete variables (label of each sample). However, in this paper, we observed that some MRF models are similar to each other if we take into account a proper selection of their parameters.

Another interesting conclusion concerns with the feature extraction model: On average, feature vector (II) has obtained slightly better recognition rates, but still similar to the results obtained with feature vector (I). Such results are very interesting since they point out that we do not need to analyze the whole neighborhood of a given sample. We can consider such information by just adding the $(x, y)$ position of each sample in the feature vector. Therefore, we can save up to $60 \%$ of computational time if we adopt feature vector II, for instance. Since Ikonos-2 MS image has a higher resolution than CBERS-2B one, its ground-truth map seems to be more complicated to be classified.

In the second round of experiments, we aimed at evaluating the feasibility of modeling the problem of fine-tuning parameter $\beta$ as a metaheuristic-based optimization problem. Tables III-VI present the mean average results for all MRF models optimized by BF, HS, PSO, NM, and GD, considering 
TABLE V

Mean Accuracy Results Considering SVM-Based Classifiers Different Markov Models Over CBERS-2B IMAGE With $\beta$ Optimized by BF, HS, and PSO. The Best Accuracy of Each Pair (Markov Model, Optimization TechniQue) Is in Bold

\begin{tabular}{|l|c|c|c|c|c|c|}
\hline Technique & Mode value & BF & HS & PSO & NM & GD \\
\hline SVM & \multicolumn{3}{|c|}{$87.93 \% \pm 0.22$} \\
\hline & - & $\mathbf{9 0 . 3 6 \%} \pm \mathbf{0 . 2 3}$ & $\mathbf{9 0 . 4 4 \%} \pm \mathbf{0 . 3 2}$ & $\mathbf{9 0 . 4 8 \%} \% \mathbf{0 . 2 2}$ & $\mathbf{9 0 . 3 5 \%} \pm \mathbf{0 . 3 5}$ & $\mathbf{9 0 . 0 7 \%} \pm \mathbf{0 . 7 4}$ \\
SVM-MRF/ & 3 & $87.09 \% \pm 0.52$ & $87.07 \% \pm 0.53$ & $87.09 \% \pm 0.54$ & $87.09 \% \pm 0.52$ & $87.06 \% \pm 0.62$ \\
Potts & 4 & $\mathbf{9 0 . 0 8 \%} \pm \mathbf{0 . 4 2}$ & $\mathbf{9 0 . 0 5 \%} \pm \mathbf{0 . 3 4}$ & $\mathbf{9 0 . 1 0 \%} \pm \mathbf{0 . 3 9}$ & $\mathbf{9 0 . 1 3 \%} \pm \mathbf{0 . 4 6}$ & $\mathbf{8 9 . 9 2 \%} \pm \mathbf{0 . 5 3}$ \\
\hline & - & $\mathbf{9 0 . 2 8 \%} \pm \mathbf{0 . 4 7}$ & $\mathbf{9 0 . 1 4 \%} \pm \mathbf{0 . 4 8}$ & $\mathbf{9 0 . 1 4 \%} \pm \mathbf{0 . 3 8}$ & $\mathbf{9 0 . 3 8 \%} \pm \mathbf{0 . 2 3}$ & $\mathbf{8 9 . 6 9 \%} \pm \mathbf{1 . 1 7}$ \\
SVM-MRF/ & 3 & $87.02 \% \pm 0.47$ & $87.02 \% \pm 0.55$ & $87.02 \% \pm 0.46$ & $87.00 \% \pm 0.48$ & $86.98 \% \pm 0.47$ \\
GIMLL-L1 & 4 & $\mathbf{8 9 . 9 8 \%} \pm \mathbf{0 . 3 2}$ & $\mathbf{8 9 . 8 7 \%} \pm \mathbf{0 . 3 9}$ & $\mathbf{8 9 . 9 2 \%} \pm \mathbf{0 . 3 1}$ & $\mathbf{8 9 . 9 4 \%} \pm \mathbf{0 . 3 8}$ & $\mathbf{8 9 . 7 7 \%} \pm \mathbf{0 . 3 8}$ \\
\hline & - & $\mathbf{9 0 . 0 6 \%} \pm \mathbf{0 . 3 5}$ & $\mathbf{9 0 . 2 4 \%} \pm \mathbf{0 . 3 1}$ & $\mathbf{9 0 . 0 5 \%} \pm \mathbf{0 . 2 6}$ & $\mathbf{9 0 . 1 8 \%} \pm \mathbf{0 . 2 0}$ & $\mathbf{8 9 . 2 8 \%} \pm \mathbf{0 . 6 5}$ \\
SVM-MRF/ & 3 & $87.00 \% \pm 0.48$ & $87.00 \% \pm 0.47$ & $86.98 \% \pm 0.46$ & $86.98 \% \pm 0.46$ & $86.94 \% \pm 0.47$ \\
GIMLL-L2 & 4 & $\mathbf{8 9 . 9 1 \%} \pm \mathbf{0 . 3 1}$ & $\mathbf{8 9 . 8 4 \%} \pm \mathbf{0 . 3 5}$ & $\mathbf{8 9 . 9 5 \%} \pm \mathbf{0 . 3 5}$ & $\mathbf{8 9 . 9 6 \%} \pm \mathbf{0 . 3 1}$ & $\mathbf{8 9 . 5 6 \%} \pm \mathbf{0 . 3 2}$ \\
\hline & - & $89.34 \% \pm 0.20$ & $\mathbf{8 9 . 2 8 \%} \pm \mathbf{0 . 7 1}$ & $\mathbf{8 9 . 3 2 \%} \pm \mathbf{0 . 2 9}$ & $\mathbf{8 8 . 8 1 \%} \pm \mathbf{0 . 9 7}$ & $\mathbf{8 8 . 1 5 \%} \pm \mathbf{1 . 0 9}$ \\
SVM-MRF/ & 3 & $86.89 \% \pm 0.51$ & $86.89 \% \pm 0.52$ & $86.90 \% \pm 0.53$ & $86.83 \% \pm 0.48$ & $86.73 \% \pm 0.37$ \\
GMRF & 4 & $\mathbf{8 9 . 3 7 \%} \pm \mathbf{0 . 2 6}$ & $\mathbf{8 9 . 4 3 \%} \pm \mathbf{0 . 2 7}$ & $\mathbf{8 9 . 3 9 \%} \pm \mathbf{0 . 2 4}$ & $\mathbf{8 9 . 1 4 \%} \pm \mathbf{0 . 5 5}$ & $\mathbf{8 9 . 0 7 \%} \pm \mathbf{0 . 3 2}$ \\
\hline
\end{tabular}

TABLE VI

Mean Accuracy Results Considering SVM-Based Classifiers DifFerent Markov Models Over IKonos-2 MS Image With $\beta$ Optimized by BF, HS, ANd PSO. The Best ACCuracy of Each Pair (Markov Model, Optimization TechniQue) Is in Bold

\begin{tabular}{|c|c|c|c|c|c|c|}
\hline Technique & Mode value & $\mathrm{BF}$ & HS & PSO & NM & GD \\
\hline SVM & & \multicolumn{5}{|c|}{$86.92 \% \pm 0.36$} \\
\hline & - & $87.88 \% \pm 0.60$ & $87.63 \% \pm 0.54$ & $87.68 \% \pm 0.67$ & $87.74 \% \pm \mathbf{0 . 6 2}$ & $87.48 \% \pm 0.55$ \\
\hline SVM-MRF/ & 3 & $87.46 \% \pm 0.37$ & $87.48 \% \pm 0.42$ & $87.43 \% \pm 0.40$ & $87.46 \% \pm 0.37$ & $87.50 \% \pm 0.34$ \\
\hline Potts & 4 & $\mathbf{8 8 . 3 0 \%} \pm \mathbf{0 . 5 6}$ & $88.25 \% \pm 0.61$ & $88.15 \% \pm \mathbf{0 . 5 1}$ & $\mathbf{8 8 . 2 1 \%} \pm \mathbf{0 . 5 2}$ & $88.12 \% \pm 0.41$ \\
\hline & - & $87.37 \% \pm 0.47$ & $87.41 \% \pm 0.52$ & $87.43 \% \pm 0.30$ & $87.61 \% \pm 0.57$ & $87.29 \% \pm 0.41$ \\
\hline SVM-MRF/ & 3 & $87.45 \% \pm 0.40$ & $87.44 \% \pm 0.39$ & $87.46 \% \pm 0.41$ & $87.41 \% \pm 0.35$ & $87.44 \% \pm 0.40$ \\
\hline GIMLL-L1 & 4 & $\mathbf{8 8 . 1 8 \%} \pm \mathbf{0 . 5 2}$ & $\mathbf{8 8 . 2 4 \%} \pm \mathbf{0 . 5 1}$ & $88.11 \% \pm 0.44$ & $88.22 \% \pm 0.52$ & $88.00 \% \pm 0.53$ \\
\hline & - & $87.63 \% \pm 0.57$ & $87.14 \% \pm 0.68$ & $87.41 \% \pm 0.43$ & $87.61 \% \pm 0.54$ & $87.33 \% \pm 0.50$ \\
\hline SVM-MRF/ & 3 & $87.46 \% \pm 0.40$ & $87.47 \% \pm 0.40$ & $87.47 \% \pm 0.40$ & $87.46 \% \pm 0.41$ & $87.44 \% \pm 0.35$ \\
\hline GIMLL-L2 & 4 & $\mathbf{8 8 . 2 4 \%} \pm \mathbf{0 . 5 2}$ & $88.20 \% \pm 0.48$ & $88.26 \% \pm 0.50$ & $\mathbf{8 8 . 2 2} \% \pm \mathbf{0 . 5 3}$ & $88.08 \% \pm 0.49$ \\
\hline & - & $87.05 \% \pm 0.58$ & $87.10 \% \pm 0.58$ & $86.75 \% \pm 0.84$ & $86.82 \% \pm 0.69$ & $86.65 \% \pm 0.25$ \\
\hline SVM-MRF/ & 3 & $87.45 \% \pm 0.44$ & $87.46 \% \pm 0.44$ & $87.44 \% \pm 0.42$ & $87.42 \% \pm 0.41$ & $87.34 \% \pm 0.39$ \\
\hline GMRF & 4 & $\mathbf{8 8 . 0 3 \%} \pm \mathbf{0 . 6 2}$ & $\mathbf{8 8 . 0 2 \%} \pm \mathbf{0 . 6 7}$ & $\mathbf{8 8 . 0 3 \%} \pm \mathbf{0 . 6 5}$ & $87.84 \% \pm 0.63$ & $87.72 \% \pm 0.47$ \\
\hline
\end{tabular}

the feature vector I extracted from CBERS-2B and Ikonos-2 MS images. ${ }^{8}$ From those tables, some conclusions can be observed: 1) all MRF models are similar to each other when we employ a more reliable parameter fine-tuning; 2) HS, PSO, $\mathrm{NM}$, and GD obtained similar results than the near-exhaustive search BF, but are faster; and 3) the proposed postprocessing approach to avoid overcorrection in high-frequency regions has been effective for both discrete and nondiscrete MRF models, but is better for the latter ones. Since Potts model was specially designed to work over discrete variables, it seems that its initial results were the best ever, i.e., they were not improved with the postprocessing technique, whereas the results of the other nondiscrete models can be further improved using the proposed approach (mainly with respect to Ikonos-2 MS image).

Figs. 3 and 4 shows some classification results using the Potts model optimized by BF for CBERS-2B and Ikonos-2 MS images, respectively. We can clearly observe the improvement of OPF-MRF regarding naïve OPF for both images. Additionally, the proposed postprocessing approach has avoided overcorrection in regions composed of nearby samples from distinct classes (top-middle regions of Fig. 4(b) for instance).

\section{CONCluding Remarks}

This letter has two main contributions: 1) to propose an optimization framework for nondiscrete Markovian models;

\footnotetext{
${ }^{8}$ Symbol "-" denotes that we did not employ the proposed postprocessing approach.
}

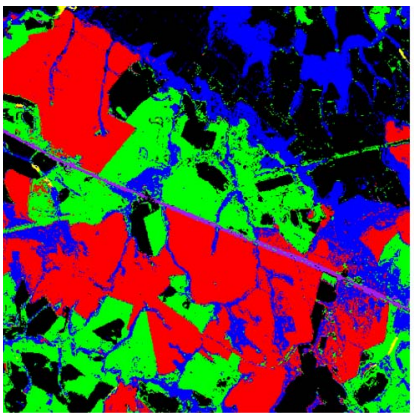

(a)

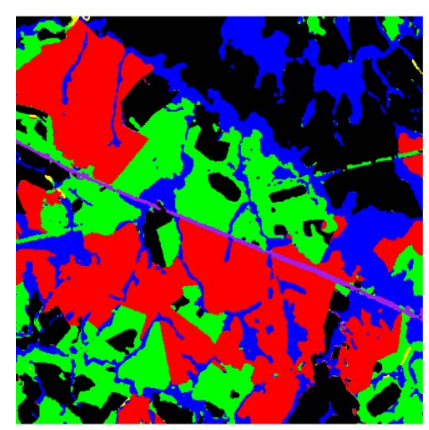

(c)

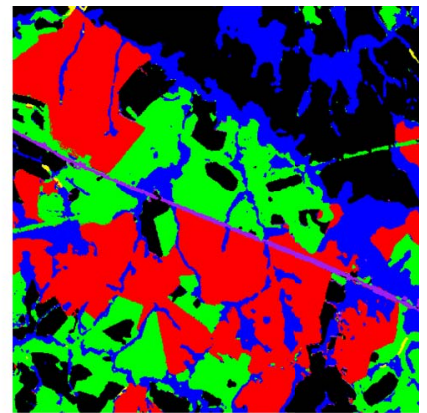

(b)

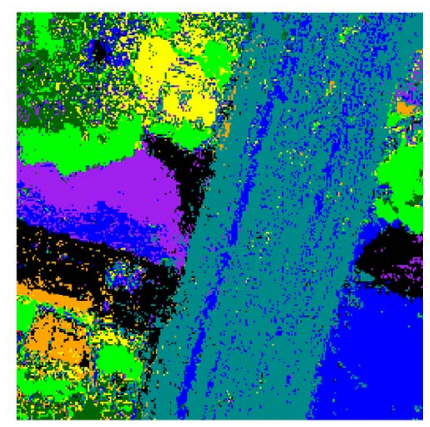

(d)
Fig. 3. Classification results over CBERS2-B image obtained by naiïve OPF using the feature extraction approach (I) in (a), OPF-MRF classification results without the postprocessing using feature extraction approach (I) in (b), and OPF-MRF classification results with the proposed postprocessing approach using feature extraction approach (I) (mode value of 5) in (c). Classification results over Ikonos-2 MS image obtained by naïve OPF using the feature extraction approach (I) in (d). 


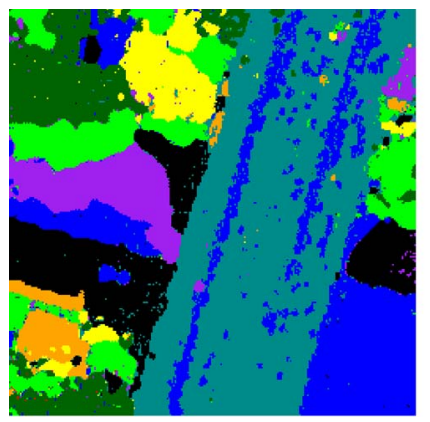

(a)

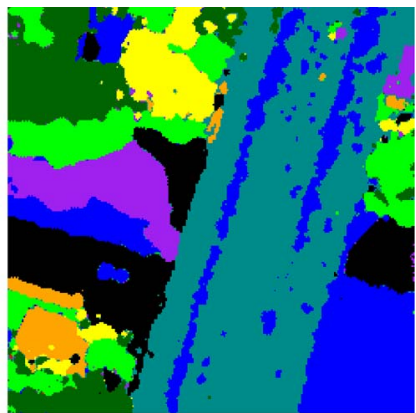

(b)
Fig. 4. Classification results over Ikonos-2 MS image obtained by OPF-MRF classification results without the postprocessing using feature extraction approach (I) in (a) and the OPF-MRF classification results with the proposed postprocessing approach (mode value of 5 in a $3 \times 3$ neighborhood system) using feature extraction approach (I) in (b).

and 2) to present a postprocessing approach to avoid overcorrections on high-frequency regions, thus obtaining improvements regarding standard OPF-MRF. The experimental section was conducted over two satellite images, demonstrating that the proposed approach can provide better results than the original OPF-MRF, and both discrete and nondiscrete MRF models may be similar to each other when we employ a proper parameter fine-tuning.

\section{ACKNOWLEDGMENT}

The authors would like to thank R. J. Pisani for providing the images.

\section{REFERENCES}

[1] A. Schmidt, J. Niemeyer, F. Rottensteiner, and U. Soergel, "Contextual classification of full waveform lidar data in the wadden sea," IEEE Geosci. Remote Sens. Lett., vol. 11, no. 9, pp. 1614-1618, Sep. 2014.

[2] Y. Tarabalka, M. Fauvel, J. Chanussot, and J. A. Benediktsson, "SVM- and MRF-based method for accurate classification of hyperspectral images," IEEE Geosci. Remote Sens. Lett., vol. 7, no. 4, pp. 736-740, Oct. 2010.

[3] G. Moser and S. B. Serpico, "Combining support vector machines and Markov random fields in an integrated framework for contextual image classification," IEEE Trans. Geosci. Remote Sens., vol. 51, no. 5, pp. 2734-2752, May 2013.

[4] P. Ghamisi, J. A. Benediktsson, and M. O. Ulfarsson, "Spectral-spatial classification of hyperspectral images based on hidden Markov random fields," IEEE Trans. Geosci. Remote Sens., vol. 52, no. 5, pp. 2565-2574, May 2014.

[5] J. P. Papa, A. X. Falcão, and C. T. N. Suzuki, "Supervised pattern classification based on optimum-path forest," Int. J. Imaging Syst. Technol., vol. 19, no. 2, pp. 120-131, Jun. 2009.

[6] J. P. Papa, A. X. Falcão, V. H. C. Albuquerque, and J. M. R. S. Tavares, "Efficient supervised optimum-path forest classification for large datasets," Pattern Recognit., vol. 45, no. 1, pp. 512-520, Jan. 2012.

[7] J. P. Papa, A. X. Falcão, G. M. Freitas, and A. M. H. Avila, "Robust pruning of training patterns for optimum-path forest classification applied to satellite-based rainfall occurrence estimation," IEEE Geosci. Remote Sens. Lett., vol. 7, no. 2, pp. 396-400, Apr. 2010.

[8] D. Osaku et al., "Improving land cover classification through contextualbased optimum-path forest," Inf. Sci., vol. 324, pp. 60-87, Dec. 2015.

[9] D. Osaku et al., "Optimizing contextual-based optimum-forest classification through swarm intelligence," in Advanced Concepts for Intelligent Vision Systems. Berlin, Germany: Springer-Verlag, 2013, pp. 203-214.

[10] D. Osaku, A. L. M. Levada, and J. P. Papa, "On the influence of Markovian models for contextual-based optimum-path forest classification," in Progress in Pattern Recognition, Image Analysis, Computer Vision, and Applications, vol. 8827. Berlin, Germany: Springer-Verlag, 2014, pp. $462-469$. 